\title{
Pharmacogenomics Trial Characteristics Domain
}

National Cancer Institute

\section{Source}

National Cancer Institute. Pharmacogenomics Trial Characteristics Domain. NCI

Thesaurus. Code C111290.

A special purpose domain that contains design parameters used in performing

pharmacogenomic assessments. 